\title{
APPLICATION OF A SMITH PREDICTOR BASED NONLINEAR PREDICTIVE CONTROLLER TO A SOLAR POWER PLANT
}

\author{
Manuel Gálvez-Carrillo ${ }^{*}$, Robin De Keyser, Clara Ionescu \\ *Department of Control Engineering and System Analysis, Université Libre de Bruxelles, \\ Av. F.D. Roosevelt 50-CP165/55, B1050 Brussels, Belgium \\ Department of Electrical energy, Systems and Automation, Ghent University, \\ Technologiepark 913, B9052 Gent, Belgium
}

\begin{abstract}
Renewable energies are gaining space in the energy generation panorama, thanks to technological advances and policy support. To take profit of these energies in an optimal and sustained way, research of new control strategies becomes imperative. This work presents the study and application of a nonlinear control strategy, where a Smith Predictor is added to the Nonlinear Extended Prediction Self-Adaptive Control (NEPSAC), for the control of a Solar Power Plant. Different simulations are performed to study the effect of the design parameters in the dynamic behavior of the system. Copyright () 2007 IFAC
\end{abstract}

Keywords: Nonlinear predictive control, model based control, solar energy, variable time delay, delay compensation.

\section{INTRODUCTION}

Apart from the 'classical' PID controller, MPC is practically the only control strategy which has also been widely adopted by industry, starting around 1990. MPC technology was originally developed for power plant and petroleum refinery control applications, but nowadays it can be found in a wide variety of manufacturing environments including chemicals, food processing, automotive, aerospace, metallurgy, mechatronics, pulp and paper, ... It has even become a de facto industry standard in the petrochemical industry due to its intrinsic capability for dealing with constraints and with multivariable systems. Recently, the control engineering research community developed the notion of NMPC: Nonlinear Model Predictive Control, where a more accurate (nonlinear) model of the system is used for prediction and optimization (Allgöwer $e t$ al, 2004).

More than ever, renewable energies are attracting nowadays a lot of attention due to the negative impact that the use of fossil fuel sources for energy has on the current global economy and environment.
Alternative sources of energy may be at present moment more expensive or more difficult to obtain. However, given the rocketing prices of gasoline and the prospect that oil resources will be depleted, the search for alternative energy sources is imperative. (Woloski, 2006). Therefore, it is necessary not only to adequately design the conversion processes, but also to ensure their optimal and economical operation by means of efficient control strategies.

The present application is a conversion system which uses the solar irradiation. The main characteristic of solar power plants is that the source of energy, the solar irradiation, cannot be manipulated and acts as a disturbance. Moreover, the process has strong nonlinear dynamics and variable dead-time, both of them due to the manipulated variable (oil-flow). The contribution of the present work is the use of a nonlinear model in order to implement a nonlinear predictive control strategy that includes a Smith Predictor to compensate for the variable time delay.

The paper is organized as follows: section II presents a description of the plant and control problem. The 
procedure used to model the process is presented in section III. The nonlinear control strategy is presented in section IV and section $\mathrm{V}$ presents the results for different simulation tests with real data inputs. A conclusion section summarizes the main outcome of this contribution.

\section{PLANT DESCRIPTION}

The system studied here is the ACUREX distributed collectors field. The PSA plant (Plataforma Solar de Almería) is based on a parabolic-trough technology, and is located in the desert of Tabernas, in the South of Spain. According to (Quaschning and Blanco, 2001), this is the only solar thermal technology with commercial operating systems. A good overview of the control problem and of different (linear) strategies is given in (Camacho et al, 1997). The field is composed of 480 modules of solar collectors, arranged in 10 parallel rows, each row consisting of 48 modules arranged in 4 groups. Each collector contains a reflecting parabolic surface that focuses the solar irradiation onto a receiver pipe, placed in the parabola focal line, in order to heat up the fluid circulating through it. The total solar collecting surface is $2672 \mathrm{~m}^{2}$ and the axis of the collectors are oriented East $\rightarrow$ West, with one axis (elevation) solar tracking system. An eagle-eye picture of the complete PSA is presented in figure 1.

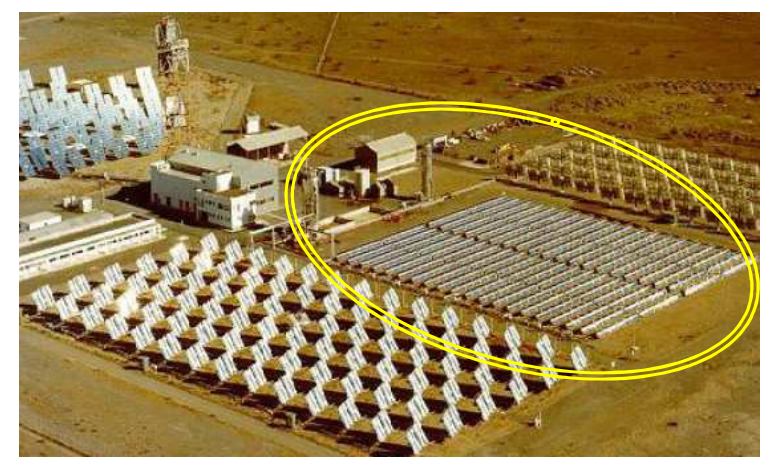

Fig. 1. Overview of the Plataforma Solar de Almería (PSA); the marked area denotes the ACUREX field.

The manipulated variable is the oil-flow rate of a thermal oil (Santotherm 55), which can be heated up to $300{ }^{\circ} \mathrm{C}$ without loosing its thermal properties. The oil is stored in a storage tank of $140 \mathrm{~m}^{3}$ capacity and is pumped from its bottom to the field and returned (stored) at the top of the tank. The oil at low (inlet) temperature is propelled by a pump, traveling trough a pipe (called supply pipe) until it reaches the collectors. A collecting pipe (called return pipe) returns the heated oil to the top of the tank. The oil properties permit stratified energy storage according to its density, allowing the use of only one tank for both cold and hot oil, the hottest oil being situated at the top of the tank. The stored energy is then transformed via a conversion system that can either be a steam turbine for generating electricity, or a desalination plant. A diagram of the ACUREX is given in figure 2 .

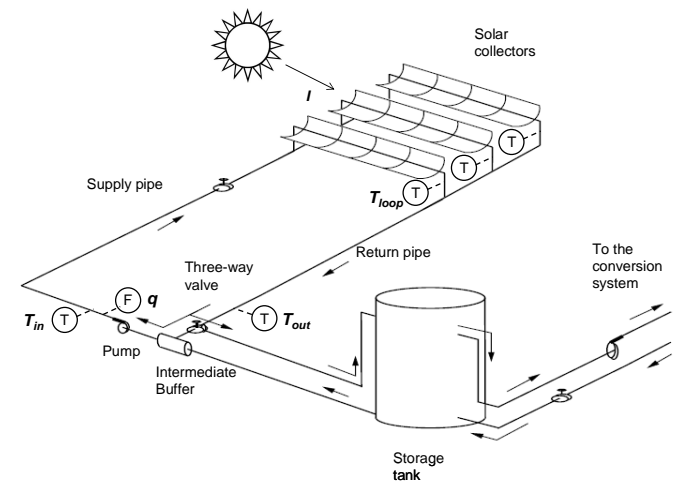

Fig. 2. Diagram of the ACUREX field

The objective of the control strategy is to ensure that the outlet temperature of the field tracks the reference signal (imposed by the inlet condition levels of the conversion system) and at the same time rejects the disturbances. The latter are mainly due to (quite fast) variations in the solar irradiation and to varying inlet oil temperature. The control is done by manipulating the oil-flow pumped into the field. There are constraints both in the manipulated variable (oil-flow varying from $2 \mathrm{l} / \mathrm{s}$ to $10 \mathrm{l} / \mathrm{s}$ ), as well as in the controlled variable (maximum outlet oil temperature equals $300^{\circ} \mathrm{C}$ and maximum difference between outlet and inlet oil temperatures equals $80^{\circ} \mathrm{C}$ ).

\section{PROCESS MODELLING}

Several nonlinear models and nonlinear control approaches for this process have been presented since the beginning of the $21^{\text {st }}$ century. Nonlinear models with concentrated parameters are presented in (Pickard, 2000), combined with an adaptive control strategy. Some approaches on nonlinear control focus on the linearization of a nonlinear control problem. For example, starting from a Partial Differential Equation (PDE) model of the plant, a design based on Lyapunov methods is proposed in (Johansen and Storaa, 2002), using internal energy as Lyapunov function. In (Silva et al, 2003), a change in time scale which linearizes the plant is implemented, allowing very high and sudden changes in the reference variable. A feedforward linearization control strategy where both feedforward and feedback can be achieved is given in (Cirre et al, 2005). Starting from nonlinear PDE, a lumped parameter model is obtained by applying Orthogonal Collocation and an Adaptive Receding Horizon Control in (Igreja et al, 2005).

When modeling a plant, a sound engineering tradeoff between complexity and accuracy is necessary. In this section, the dynamic behavior of the solar collector field is presented, followed by the modeling of the variable dead time.

\subsection{Dynamic behavior of the solar collector field}

The energy balance for a differential control volume of length $d x$ can be presented by partial differential equations for the metal tube and for the fluid, respectively: 


$$
\begin{gathered}
\rho_{m} C_{m} A_{m} \frac{\partial T_{m}}{\partial t}=I n_{o} G-H_{l} G\left(T_{m}-T_{a}\right)-D H_{t}\left(T_{m}-T_{f}\right)^{(1)} \\
\rho_{f} C_{f} A_{f} \frac{\partial T_{f}}{\partial t}+\rho_{f} C_{f} q \frac{\partial T_{f}}{\partial x}=D H_{t}\left(T_{m}-T_{f}\right)
\end{gathered}
$$

where $m$ refers to metal and $f$ refers to fluid. The other parameters are: $\rho:$ density $\left(\mathrm{kg} / \mathrm{m}^{3}\right), C:$ specific heat $\left(\mathrm{J} / \mathrm{kg}{ }^{\circ} \mathrm{C}\right), \quad$ A : transversal area $\left(\mathrm{m}^{2}\right), T$ : temperature $\left({ }^{\circ} \mathrm{C}\right), T_{a}$ : ambient temperature $\left({ }^{\circ} \mathrm{C}\right), I$ : solar irradiation $\left(\mathrm{W} / \mathrm{m}^{2}\right), n_{o}$ : optical efficiency, $H_{l}$ : global coefficient of thermal losses $\left(\mathrm{W} / \mathrm{m}^{2}{ }^{\circ} \mathrm{C}\right), H_{t}$ : coefficient of metal-fluid transmission $\left(\mathrm{W} / \mathrm{m}^{2}{ }^{\circ} \mathrm{C}\right), G$ : collector aperture $(\mathrm{m}), D$ : inner diameter of the pipe (m) and $q$ : oil-flow $\left(\mathrm{m}^{3} / \mathrm{s}\right)$.

Dead-time appears when mass or energy transportation is involved. Unlike ordinary differential equations (ODE), these systems are infinite-dimensional in nature and dead-times are often a source of instability (Fridman, 2003). The time during which the oil travels through the pipes is a function of the varying flow-rate, thus a variable dead-time is present and a model is needed to capture its changes. During normal operation, the dead-time $\tau_{d}$ has to be calculated as the upper limit of the integral from (3). Hence, it calculates the distance that a particle of fluid travels in the pipe as being:

$$
L=\int_{0}^{\tau_{d}} v(\tau) d \tau \rightarrow L=\frac{T_{s}}{A} \sum_{j=1}^{j=d} q(j)
$$

where $A$ is the constant cross-section of the pipe, $T_{s}$ is the sampling time, $q(j)$ the oil-flow, calculated at each sample $j$. In a discrete control system, where the flow changes at every sampling time, the dead time can be approximated as the number $d$ of sampling periods that the flow needs to travel along the length $L$ of the pipe. From (3) results:

$$
\sum_{j=t-d}^{j=t} q(j)=\frac{A L}{T_{s}}
$$

The objective is thus to calculate the number of samples $d$ as a function of the past oil-flows until the volume of the pipe is filled, resulting in $\tau_{d} \approx d \cdot T_{s}$.

\subsection{Nonlinear model of the process}

Since there are 10 loops, simulating each of them separately will increase the complexity of the model. Modeling one characteristic loop will be representative for the dynamics of the complete solar field. The system is conceptually divided in a supply pipe, a heated pipe (solar collector field) and return pipe, as in figure 3. For simplicity, only the dynamics of the fluid are used, by combining (1) and (2) and neglecting the dynamics of the metal tube. The resulting model (figure 3) is a grey-box model, based on physical principles with some identified parameters.

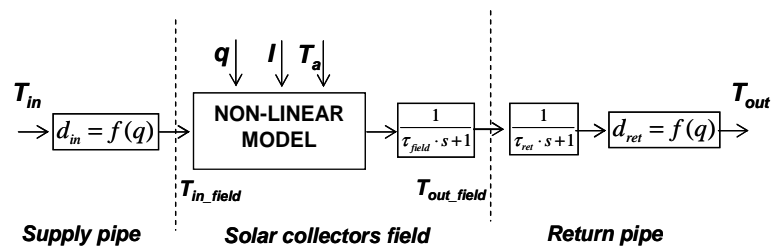

Fig. 3. Scheme of the nonlinear model

The block "Non-linear model" accounts for the dynamics of the solar collector field from (5). This is the model used for simulating the real-plant (called "Plant" in the schemes below). The model described by (6) is used for prediction (called "Non-linear model" in the nonlinear control strategy). These models can be either distributed (with different number of partitions) or lumped, depending on the adjustment in the length of the partition $\Delta x$. The time-dependent ODE equations applied to the $n^{\text {th }}$ partition are:

$$
\begin{gathered}
\rho C A \frac{d T_{n}}{d t}=k_{\text {plant }} \cdot G \quad I- \\
\rho C q \frac{T_{n}-T_{n-1}}{\Delta x}-H_{l} D\left(T_{n}-T_{a}\right) \\
\rho C A \frac{d T_{n}}{d t}=k_{\text {model }} G I-\rho C q \frac{T_{n}-T_{n-1}}{\Delta x}
\end{gathered}
$$

Neglecting the heat losses provides un-modeled dynamics and offers low-computational effort. For a given discrete time $j$, the inputs are the oil-flow $q(j)$ and the measured solar irradiation $I(j)$, the ambient temperature $T_{a}(j)$ and the delayed inlet oil temperature $T_{i n}\left(j-d_{i n}\right)=T_{\text {in_field }}(j)$ that constitutes the model for the supply pipe.

There are two consequences resulting from neglecting the dynamics of the metal tube. If the real values of the optical efficiency $n_{o}$ will be included in (5) or (6), higher values of temperature will result than those obtained in the real case. For that reason, the value of the optical efficiency will be represented by the tuning parameter $k$, where $k<n_{o}$ and is one parameter that has to be identified. The second consequence is that without the metal tube dynamics, the heating process of the oil will be faster than in the real case. To overcome this fact, the addition of extra (first-order) dynamics is used for both supply pipe and solar collector field model:

$$
\frac{1}{\tau \cdot s+1}, \tau=\frac{M}{q}
$$

where the time constants depend on the inverse of the oil-flow and $M$ is a tuning parameter. Finally, the return pipe is modeled as a combination of a first order system plus a calculation of the dead-time as presented in (7) and (4) respectively.

\section{NONLINEAR CONTROLLER}

NMPC refers to MPC schemes that are based on nonlinear models and/or consider a non-quadratic 
cost-function and nonlinear constraints (Allgöwer et $a l, 2004)$. The key-concept of NEPSAC (Nonlinear EPSAC) is to use directly a nonlinear model of the true process, without any linearization involved in the control strategy (De Keyser, 2003).

\subsection{The NMPC formulation.}

The objective of the non-linear model predictive control (NMPC) is to find the input sequence that minimizes a given cost function, based on a desired output trajectory over a prediction horizon $\left(N_{l} \ldots N_{2}\right)$. This cost function considers the errors between the predicted model outputs $y(t+j \mid \mathrm{t})$ and the reference trajectory $r$. It also includes the change in the input $u$, over $N_{u}$ samples (control horizon), where $N_{u} \leq N_{2}$ and $u(t+j \mid \mathrm{t})=u\left(t+N_{u}-1 \mid \mathrm{t}\right)$ for $\mathrm{j}>=N_{u}$. These errors are normally weighted $(\gamma, \lambda)$, resulting in:

$$
\begin{aligned}
& \min _{u(t) \ldots u\left(t+N_{u}-1\right)} J=\sum_{j=N_{1}}^{N_{2}} \gamma[y(t+j \mid t)-r(t+j)]^{2} \\
& +\sum_{j=0}^{N_{u}-1} \lambda[\Delta u(t+j \mid j)]^{2}
\end{aligned}
$$

where the minimization is done subject to constraints on the manipulated and controlled variables:

$$
\begin{aligned}
& y_{\min } \leq y(t+j \mid t) \leq y_{\max } \quad k=1 \ldots N_{2} \\
& u_{\text {min }} \leq u(t+j \mid t) \leq u_{\max } \quad k=0 \ldots N_{u}-1 \\
& \Delta u_{\min } \leq \Delta u(t+j \mid t) \leq \Delta u_{\max } \quad k=0 \ldots N_{u}-1
\end{aligned}
$$

From the calculated input sequence, only the first sample is applied to the process. The entire procedure is repeated at the next sampling moment, according to the receding horizon strategy. Due to the nonlinear model and constraints, there is no direct analytical solution as in the linear case, resulting in a nonlinear optimization problem. Depending on the model and the constraints, this can be time consuming and difficult to implement in real time.

\subsection{The NEPSAC formulation.}

The Nonlinear EPSAC algorithm starts from the fact that the superposition principle involved in the original EPSAC algorithm (De Keyser, 2003) does not hold for non-linear models. Therefore, it develops a computationally friendly iterative solution to overcome this problem. Consider the process model:

$$
y(t)=x(t)+n(t)
$$

where $x(t)$ is a generic dynamic model:

$$
x(t)=f[x(t-1), x(t-2), \cdots, u(t-1), u(t-2), \cdots]
$$

and $n(t)$ is the disturbance process model, that can be expressed in its simple form as:

$$
n(t)=\frac{C\left(q^{-1}\right)}{D\left(q^{-1}\right)} e(t)
$$

where $e(t)$ is a white noise. The predicted values of the output are calculated by:

$$
y(t+j \mid t)=x(t+j \mid t)+n(t+j \mid t)
$$

The EPSAC strategy considers that the future response can be expressed as the cumulative result of two effects: a base response that accounts for the effect of past control, a base future control scenario and the effect of future disturbances, and an optimizing response that accounts for the effect of the optimizing future control actions. That means that for $j=N_{1} \ldots N_{2}$ the predicted outputs will be:

$$
y(t+j \mid t)=y_{\text {base }}(t+j \mid t)+y_{\text {opt }}(t+j \mid t)
$$

depending on the control actions, for $j=0 \ldots N_{u^{-}}-1$ :

$$
u(t+j \mid t)=u_{\text {base }}(t+j \mid t)+\delta u(t+j \mid t)
$$

The key EPSAC equation is obtained:

$$
\mathbf{Y}=\mathbf{Y}_{b a s e}+\mathbf{Y}_{\text {opt }}=\overline{\mathbf{Y}}+\mathbf{G U}
$$

with

$$
\begin{aligned}
\mathbf{Y} & =\left[y\left(t+N_{1} \mid t\right) \cdots y\left(t+N_{2} \mid t\right)\right]^{T} \\
\overline{\mathbf{Y}} & =\left[y_{\text {base }}\left(t+N_{1} \mid t\right) \cdots y_{\text {base }}\left(t+N_{2} \mid t\right)\right]^{T} \\
\mathbf{U} & =\left[\delta u(t \mid t) \cdots \delta u\left(t+N_{u}-1 \mid t\right)\right]^{T} \\
\mathbf{G} & =\left[\begin{array}{cccc|c}
h_{N_{1}} & h_{N_{1}-1} & \cdots & h_{N_{1}-N_{u}+2} & g_{N_{1}-N_{u}+1} \\
h_{N_{1}+1} & h_{N_{1}} & \cdots & \cdots & \cdots \\
\vdots & \vdots & \vdots & \vdots & \vdots \\
h_{N 2} & h_{N_{2}-1} & \cdots & h_{N_{2}-N_{u}+2} & g_{N_{2}-N_{u}+1}
\end{array}\right]
\end{aligned}
$$

and $h_{j} / g_{j}$ being the coefficients of the impulse / step response of the process in the actual operating point. The cost function in (8) can now be expressed as a quadratic form in $\mathrm{U}$ and together with the constraints (9) it is being solved using simple quadratic programming $(\mathrm{QP})$ techniques.

However, for the nonlinear case, the superposition principle expressed in (14) does not hold anymore. The NEPSAC approach consists in transforming the nonlinear optimization problem into consecutive QP problems. At each sampling step, the procedure presented above is repeated iteratively by the simple redefinition of $u_{\text {base }}(t+j \mid \mathrm{t}) \equiv u(t+j \mid \mathrm{t})$, that means that the base control policy in a given iteration step is set equal to the optimal input sequence calculated in the previous iteration step. This iteration is continued until $\delta u(t+j \mid \mathrm{t}) \approx 0$, such that $y_{\text {opt }}(t+j \mid \mathrm{t}) \approx 0$, superposition thus being eliminated. In order to accelerate the convergence, the iteration can be started setting $u_{\text {base }}(t+j \mid \mathrm{t}) \equiv u^{*}(t+j \mid \mathrm{t}-1)$, where $u^{*}$ is the optimal input sequence calculated at the previous sampling moment. A comparison of the good 
performance of the NEPSAC against other NMPC algorithms can be found in (Rueda et al, 2005).

\subsection{The Smith Predictor based NEPSAC.}

An alternative for controlling processes that present long (and varying) dead-times is the use of a DeadTime Compensator (DTC). The Smith Predictor (SP) was the first control system proposed in the literature that included a DTC and is perhaps the best known DTC in industrial applications.

The combination of MPC with DTC is an idea presented in (Normey-Rico, 1999) where a filtered SP combined with the GPC algorithm - a linear MPC strategy - is studied in detail. However, it has not been applied to the NMPC. The combination of the filtered SP with the NEPSAC, namely Smith Predictor based NEPSAC (SP-NEPSAC), provides a control strategy for nonlinear processes with variable dead-times. The resulting structure is presented in figure 4 .

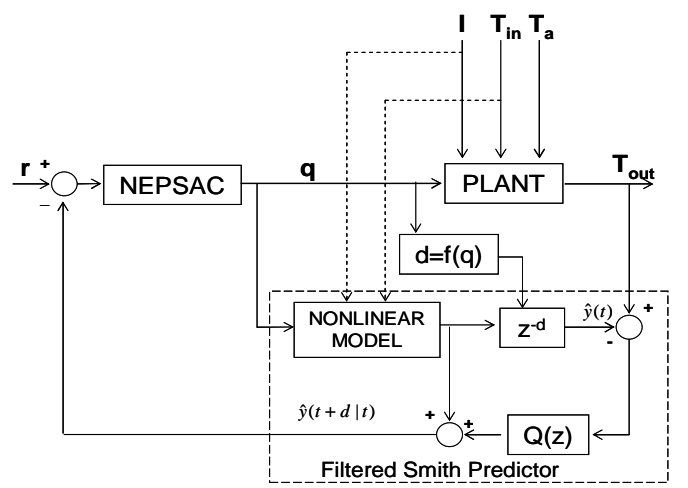

Fig. 4. SP-NEPSAC control scheme.

The addition of the filtered SP has two main advantages. The feedback signal is equal to the open loop response of the nonlinear model presented in figure 3 without the output dead-time, plus the filtered model error. Therefore, the first advantage is that prediction horizons $N_{1}, N_{2}$ are no longer dependent on the value of the dead-time, being $N_{1}=1$ and $N_{2}=N$. Especially for longer and varying dead-times, this reduces considerably the computational load of a predictive controller. The second advantage is that the robustness of the system is increased against modeling errors (present due to varying dead-time).

\section{SIMULATION RESULTS}

The plant is represented by a distributed parameters model of 10 partitions using (5) for the solar field dynamics. First, a nominal operating point is chosen and then the real-life disturbances are applied. The sampling time is set to $T_{s}=30 \mathrm{~seconds}$. The nominal values are: flow: $q_{\text {nom }}=6 \mathrm{l} / \mathrm{s}$; outlet temperature: Tout $_{\text {nom }}=220,9{ }^{\circ} \mathrm{C}$; solar irradiation: $I_{\text {nom }}=900$ $\mathrm{W} / \mathrm{m}^{2}$, inlet temperature : $\operatorname{Tin}_{\text {nom }}=155^{\circ} \mathrm{C}$; ambient temperature : $T a_{\text {nom }}=32,5^{\circ} \mathrm{C}$. Figure 5 presents the real-life disturbances applied to the system.
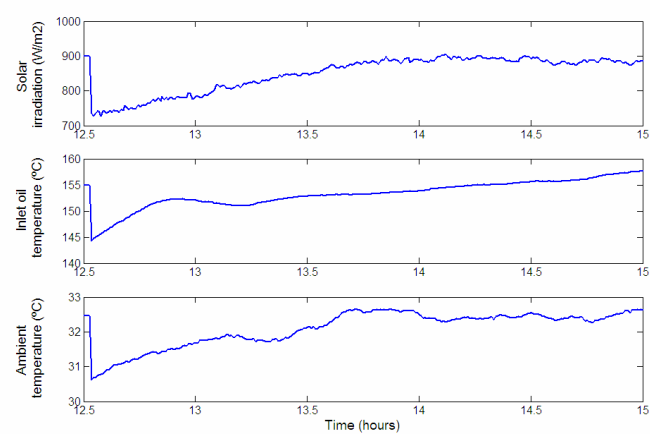

Fig. 5. The present disturbances acting on the system.

Test 1: Choosing a model for prediction. Defining $n$ as the number of partitions of the field pipe model, the prediction model is varied from a lumped parameters model $(n=1)$ to a distributed parameters model with ( $n=3$ and $n=5)$. The other parameters where $N=10, N_{u}=1, \gamma=1$, and $\lambda=0$. Figure 6 depicts a comparison between the prediction models.

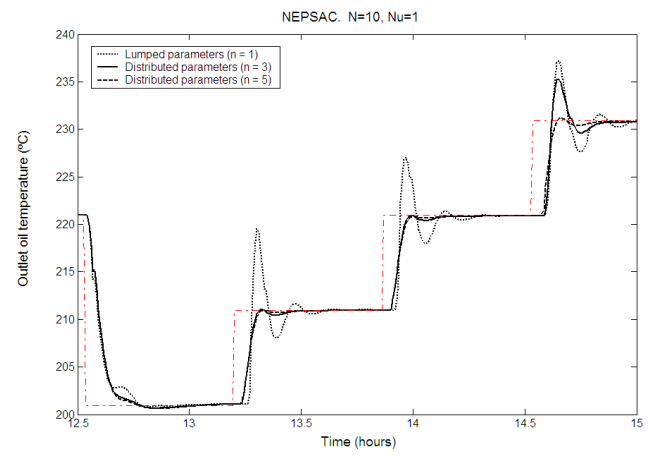

Fig. 6. Performance with different models for prediction.

Test 2: Tuning the control horizon. Three different tests have been made for $\mathrm{N}=\{8 ; 10 ; 15\}$. A distributed parameters model $(n=5)$ has been used for prediction, with $N_{u}=1, \gamma=1$, and $\lambda=0$, depicted by figure 7 . The overall performance is substantially improved when changing from 8 to 10 samples, but varies less when changing from 10 to 15 samples. Hence, $N=$ 10 seems a reasonable choice for this controller.

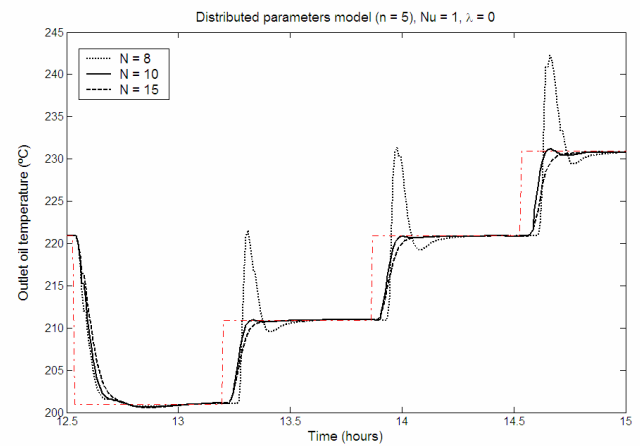

Fig. 7. Performance with different prediction horizons. Distributed parameters model $(n=5)$

Test 3: Tuning the control effort penalization. Three different tests have been made with the lumped parameters model and $N=10, N_{u}=1, \gamma=1$. From figure 8 can be observed that the penalization $\lambda=\{0$; $5 ; 50\}$ has to suffer a big increment in order to have 
an effect. A trade-off between overshoot and settling time must be made. The effect on a distributed parameters model is almost unnoticeable if $N_{u}=1$.

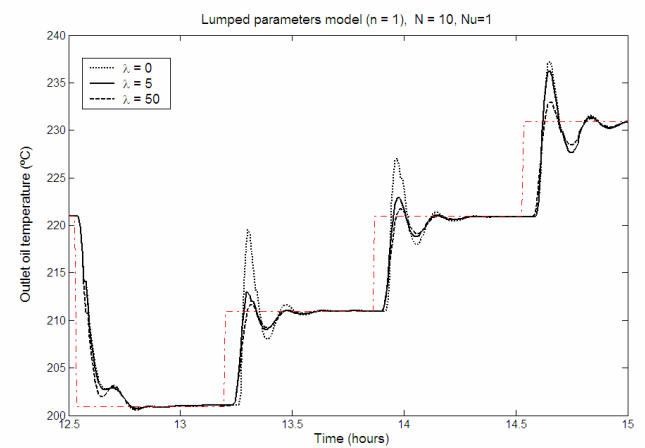

Fig. 8. Performance with control horizon $N_{u}=1$.

In the case of $N=10, N_{u}=2$ and $\gamma=1$, the use of the lumped parameters model gave very oscillatory response. Therefore, a distributed parameters model has to be used, with the slight variation of the parameter $\lambda$ having a great impact on the performance of the response. Figure 9 shows the results for $\lambda=\{1 ; 5\}$ with a model with 5 partitions.

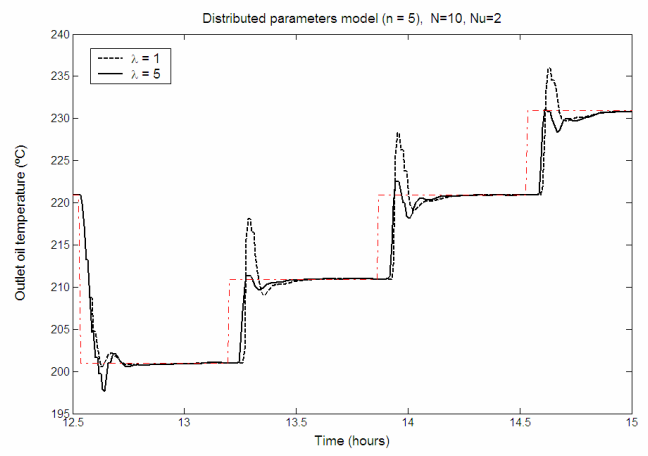

Fig. 9. Performance with control horizon $N_{u}=2$.

Thus, a good design is one based on a model with 5 partitions. This design is compared in figure 10 with a Smith Predictor based EPSAC design, that includes a nonlinear feedforward compensator for the disturbances. Both designs have $N=10, N_{u}=1, \lambda=0$. The SP-NEPSAC presents the best performance.

\section{CONCLUSIONS}

The present work has presented a combination of a DTC with a NMPC algorithm. It resulted in a Smith Predictor based NEPSAC. The latter proved to be a control strategy that can handle both significant nonlinear dynamics and variable dead-times. For the PSA application, such performance is possible due to a nonlinear model based on first principles and partially tuned with extra dynamics.

Different tests have been carried out in order to analyze the influence of the prediction model and its design parameters in the performance of the overall closed-loop response. From these tests can be concluded that the use of a distributed parameters model for prediction with 5 partitions, $N=10, N_{u}=1$ and $\lambda=0$ produces very good results.

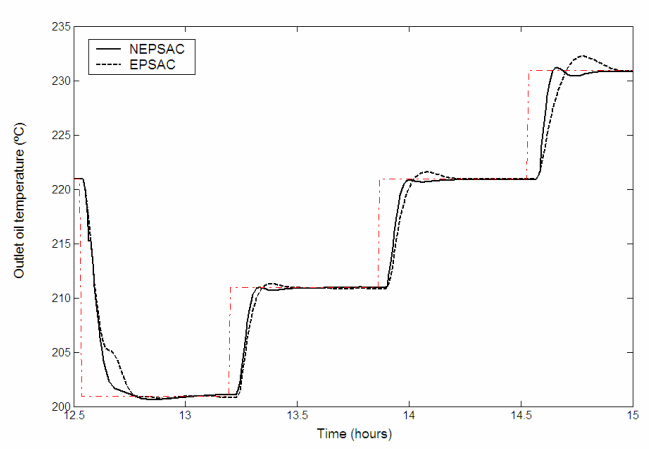

Fig. 10. Comparing SP-NEPSAC vs. SP-EPSAC.

However, if the control horizon is increased, (for example to $N_{u}=2$ ) a better model in the form of a distributed parameters model in combination with penalization on the control effort is needed in order to obtain better results.

\section{ACKNOWLEDGEMENTS}

This work was supported by a grant of the ALFA (Latin America Academic Training) Program "Process and Systems Engineering" of the European Union with reference II-0385-FA. The authors are thankful to the friendly support provided by the staff of the Plataforma Solar de Almería.

\section{REFERENCES}

Allgöwer, F., R. Findeisen and Z.K. Nagy (2004). Nonlinear Model Predictive Control: From Theory to Application. J. Chin. Inst. Chem. Engrs., 35, 3, pp. 299-315.

Camacho, E. F., M. Berenguel, and F. Rubio (1997). Advanced Control of Solar Power Plants, Springer-Verlag.

Cirre, C., L. Valenzuela, M. Berenguel and E.F. Camacho (2005). Feedback Linearization Control for a Distributed Solar Collector Field. In CD-Proc. of the $16^{\text {th }}$ IFAC World Congress, Prague.

De Keyser, R. (2003). Model Based Predictive Control. In: UNESCO Encyclopaedia of Life Support Systems (EoLSS); Article contribution 6.43.16.1, Eolss Publishers Co Ltd, Oxford, ISBN 09542989 18-26-34 (www.eolss.net), 30p.

Fridman, E. and U. Shaked (2003). Special issue on time-delay systems. Int. J. Robust Nonlinear Control, 12, pp. 791-792.

Igreja, J.M., J.M. Lemos and R. N. Silva (2005). Adaptive Receding Horizon Control of a Distributed Collector Solar Field. In Proc. of the $44^{\text {th }}$ IEEE Conf. on Decision and Control, Seville, pp. 1282-1287.

Johansen, T.A. and C. Storaa (2002). Energy-based control of a solar collector field, Automatica, 38, pp.1191-1199.

Normey-Rico, J.E. (1999). Predicción para Control, Doctoral Thesis, Universidad de Sevilla, Spain.

Pickard, R. (2000). Nonlinear modelling and adaptive predictive control of a solar power plant. Control Eng. Practice, 8, pp. 937-947.

Quaschning, V., and M. Blanco (2001). Solar Power Photovoltaics or Solar Thermal Power Plants? VGB Congress Power Plants 2001, Brussels.

Rueda, A., S. Cristea, C. de Prada and R. De Keyser (2005). Nonlinear Predictive Control for a Distillation Column. In Proc. of the $44^{\text {th }}$ IEEE Conf. on Decision and Control, Seville, pp. $5156-5161$.

Silva, R.N., J.M. Lemos and L.M. Rato (2003). Variable Sampling Adaptive Control of a Distributed Collector Solar Field. IEEE Trans. Control Syst. Techn., 11, 5, pp. 765-772.

Woloski, A. (2006) Fuel of the Future: A Global Push Toward New Energy. Harvard International Review, 27, 4, pp. 40-43. 